The Bulletin of Symbolic Logic

Volume 7, Number 3, Sept. 2001

\title{
IN MEMORIAM: JOSEPH R. SHOENFIELD
}

$1927-2000$

Joseph R. Shoenfield, who made contributions of basic importance to several areas of mathematical logic and was a renowned expositor of the field, died on November 15, 2000, in Durham, North Carolina, at the age of 73. He was president of the Association for Symbolic Logic from 1972 until 1976. He delivered the Gödel Lecture at the ASL annual meeting in Durham, North Carolina in 1992.

Shoenfield's main area of research was recursion theory, and his work in this field encompassed a broad area, including degrees of unsolvability, the lattice of recursively enumerable sets, hierarchy theory, functionals, and recursion in higher types. He made important contributions to set theory, including the Shoenfield absoluteness theorem and a simplified approach to the forcing method. In model theory he developed elegant algebraic methods for showing quantifier elimination.

Shoenfield became president of the ASL in 1972 upon Dana Scott's resignation, at a time when the National Science Foundation had recently stopped funding the reviews operation of the JSL. The loss of NSF funding coupled with high expenses put the ASL into a situation where it was rapidly heading towards bankruptcy. Under Shoenfield's leadership, painful but necessary reductions in the reviews operation were made so that the budget deficit was brought under control. For this reason the continuing existence of the ASL is due in large part to his leadership.

He was also a leading expositor of mathematical logic. His book Mathematical Logic [5] is a classic work, with an original and very clear presentation of the main results in the field, and a wealth of challenging exercises. Lou van den Dries said of it: "Studying this book and doing the exercises made me a logician." Furthermore, Shoenfield wrote two books on recursion theory ([6], [14]) and a number of expository articles on recursion theory ([13], [16]) and set theory ([7], [10], [12]) which are models of clarity.

His somewhat severe manner masked a kind and caring person with a dry sense of humor. He was willing to fight passionately for causes he believed in. He treated his friends and colleagues to excellent gourmet meals which he cooked himself. He was a ferocious bridge and chess player. Indeed, when he was a graduate student at the University of Michigan, the members of his oral exam committee wondered how he could be ready to take the exam 
because they had only seen him playing bridge. It turned out he could not only answer their questions correctly but also knew the latest literature in detail. He then went back to playing bridge but deposited his thesis a year later. He joined the Department of Mathematics of Duke University in 1952 and remained there until his retirement in 1992, chairing the department from 1970 until 1973 . He remained scientifically active until shortly before his death and spoke on the history of the ASL at the 2000 ASL Annual Meeting in Urbana. He is survived by a sister.

Some aspects of Shoenfield's work are discussed below.

In his early paper [1] he proved the result, now known as the Shoenfield Limit Lemma, which characterizes the functions computable from the halting problem as those functions $f$ of the form $f(n)=\lim _{s} g(n, s)$ with $g$ recursive. This has been the fundamental tool for studying the degrees below $\mathbf{0}^{\prime}$ since that time. He also showed that there are degrees below $\mathbf{0}^{\prime}$ which are not r. e. and proved a forerunner of the Sacks Jump Theorem.

Shoenfield was the first person after Richard Friedberg and A. A. Muchnik, the inventors of the priority method, to apply this method. Indeed, in his 1961 paper [3] he invented the infinite injury priority method and used it to prove that there is an axiomatizable consistent theory of degree less than $\mathbf{0}^{\prime}$ in which every recursive function is representable. The proof yields a version of the "thickness lemma" (see Chapter VIII of Robert Soare's book [17]) and implicitly shows the existence of an incomplete high r. e. degree. A more powerful version of the infinite injury priority method was discovered independently and a bit later by Gerald Sacks and used by him to prove numerous results on the structure of the recursively enumerable degrees, culminating in the Density Theorem.

As indicated above, Shoenfield was very interested in connections between recursion theory and other areas of logic. He showed in [2] that every consistent axiomatizable theory has a complete extension of degree strictly less than $\mathbf{0}^{\prime}$. This was among the first papers on $\Pi_{1}^{0}$ subsets of $2^{\omega}$.

Shoenfield showed in [11], by a proof of almost magical elegance, that every non-low $_{2}$ r. e. degree a contains an r. e. set $A$ such that $A$ is not contained in any maximal set. As A. H. Lachlan had previously shown that every coinfinite r. e. set of low 2 degree is contained in a maximal set, Shoenfield's result completed a remarkable characterization of the degrees of coinfinite r. e. sets not contained in any maximal set as the non $\operatorname{low}_{2} r$. e. degrees.

Shoenfield also made important contributions to set theory. In "The Axioms of Set Theory" [12] he developed a cogent justification for the axioms of Zermelo-Fraenkel set theory, based on constructing the universe of sets in stages. His paper "Unramified forcing" [9] is a presentation of the method of forcing which is remarkable for its simplicity and elegance and is still frequently used to teach the forcing method. The famous Shoenfield Absoluteness Theorem [4] asserts that $\Sigma_{2}^{1}$ sentences of second-order number 
theory with constructible real parameters are absolute for the class of constructible sets $L$. In addition, he wrote well-known expository papers on measurable cardinals [7] and on Martin's Axiom [10].

In model theory, Shoenfield developed algebraic criteria for a first-order theory to admit elimination of quantifiers. Applying these criteria is often far less tedious than a direct approach. Shoenfield used such a criterion in the chapter of model theory of his book [5] to give short proofs that the theories of algebraically closed fields and of real closed fields admit elimination of quantifiers. In [8], he gave a necessary and sufficient condition for a theory $T$ to admit elimination of quantifiers, using extendibility of isomorphisms between substructures of models of $T$ and the notion of saturation.

Shoenfield had a keen sense of the history of logic and wrote a masterful appreciation of the work of S. C. Kleene [15].

CARL G. JOCKUSCh, JR.

\section{REFERENCES}

[1] J. R. Shoenfield, On degrees of unsolvability, Annals of Mathematics, vol. 69 (1959), no. 2 , pp. 644-653.

[2] — - Degrees of models, The Journal of Symbolic Logic, vol. 25 (1960), pp. 233-237.

[3] - Undecidable and creative theories, Fundamenta Mathematicae, vol. 49 (1960/1961), pp. 171-179.

[4] - The problem of predicativity, Essays on the foundations of mathematics, Magnes Press, Hebrew University, Jerusalem, 1961, pp. 132-139.

[5] — Mathematical logic, Addison-Wesley Publishing Co., Reading, Massachusetts, 1967, reprinted 2001 by the Association for Symbolic Logic.

[6] — Degrees of unsolvability, dedicated to S. C. Kleene, North-Holland Mathematics Studies, no. 2, North-Holland Publishing Co., Amsterdam-London, American Elsevier Publishing Co., New York, 1971.

[7] - Measurable cardinals, Logic colloquium '69 (Proceedings of the Summer School and Colloqium, Manchester, 1969), North-Holland, Amsterdam, 1971, pp. 19-49.

[8] - A theorem on quantifier elimination, Symposia mathematica, vol. 5, INDAM, Rome, 1969/70, Academic Press, London, 1971, pp. 173-176.

[9] - Unramified forcing, Axiomatic set theory, Proceedings of Symposia in Pure Mathematics, vol. XIII, Part I, University of California, Los Angeles, California, American Mathematical Society, Providence, Rhode Island, 1971, pp. 357-381.

[10] — Martin's axiom, American Mathematical Monthly, vol. 82 (1975), pp. 610 617.

[11] — Degrees of classes of RE sets, The Journal of Symbolic Logic, vol. 41 (1976), pp. $695-696$.

[12] - The axioms of set theory, Handbook of mathematical logic (J. Barwise, editor), Studies in Logic and Foundations of Mathematics, vol. 90, North-Holland, Amsterdam, 1977, pp. 321-344.

[13] - Nonbounding constructions, Annals of Pure and Applied Logic, vol. 50 (1990), pp. 191-205.

[14] — Recursion theory, Lecture Notes in Logic, vol. 1, Springer-Verlag, Berlin, 1993. 
[15] — The mathematical work of S. C. Kleene, this Bulletin, vol. 1 (1995), pp. 9 43.

[16] - Priority constructions, Dedicated to the late Stephen Cole Kleene, Annals of Pure and Applied Logic, vol. 81 (1996), pp. 115-123.

[17] R. I. SOARE, Recursively enumerable sets and degrees, Springer-Verlag, Berlin, Heidelberg, New York, 1987. 